\title{
Contralateral transmaxillary corridor: an augmented endoscopic approach to the petrous apex
}

\author{
Chirag R. Patel, MD,,3 Eric W. Wang, MD,1,3 Juan C. Fernandez-Miranda, MD, ${ }^{2,3}$ \\ Paul A. Gardner, MD, ${ }^{2,3}$ and Carl H. Snyderman, MD, MBA ${ }^{1,3}$

\begin{abstract}
Departments of ${ }^{1}$ Otolaryngology and ${ }^{2}$ Neurological Surgery, University of Pittsburgh School of Medicine; and ${ }^{3}$ Center for Cranial Base Surgery, University of Pittsburgh Medical Center, Pittsburgh, Pennsylvania
\end{abstract}

\begin{abstract}
OBJECTIVE The endoscopic endonasal approach (EEA) has been shown to be an effective means of accessing lesions of the petrous apex. Lesions that are lateral to the paraclival segment of the internal carotid artery (ICA) require lateralization of the paraclival segment of the ICA or a transpterygoid infrapetrous approach. In this study the authors studied the feasibility of adding a contralateral transmaxillary (CTM) corridor to provide greater access to the petrous apex with decreased need for manipulation of the ICA.
\end{abstract}

METHODS Using image guidance, EEA and CTM extension were performed bilaterally on 5 cadavers. The anterior wall of the sphenoid sinus and rostrum were removed. The angle of the surgical approach from the axis of the petrous segment of the ICA was measured. Five illustrative clinical cases are presented.

RESULTS The CTM corridor required a partial medial maxillectomy. When measured from the axis of the petrous ICA, the CTM corridor decreased the angle from $44.8^{\circ} \pm 2.78^{\circ}$ to $20.1^{\circ} \pm 4.31^{\circ}$, a decrease of $24.7^{\circ} \pm 2.58^{\circ}$. Drilling through the CTM corridor allowed the drill to reach lateral aspects of the petrous apex that would have required lateralization of the ICA or would not have been accessible via EEA. The CTM corridor allowed us to achieve gross-total resection of the petrous apex region in 5 clinical cases with significant paraclival extension.

CONCLUSIONS The CTM corridor is a feasible extension to the standard EEA to the petrous apex that offers a more lateral trajectory with improved access. This approach may reduce the risk and morbidity associated with manipulation of the paraclival ICA.

https://thejns.org/doi/abs/10.3171/2017.4.JNS162483

KEY WORDS petrous apex; cholesterol granuloma; chordoma; endoscopic endonasal surgery; transmaxillary; skull base; surgical technique

$\mathrm{T}$ HE petrous apex is one of the most difficult areas of the skull base to access. It is surrounded by multiple neurovascular structures that create obstacles and limitations for any given approach. Traditional lateral approaches include the middle fossa approach and transmastoid approaches. The middle fossa approach requires temporal lobe retraction, with associated risk of injury and/or seizure. Transmastoid approaches are limited by the cochlea, labyrinth, and facial nerve, which put hearing, balance, and facial nerve function at risk. The position of the internal carotid artery (ICA) and jugular bulb can also limit access with the infralabyrinthine or infracochlear approach. ${ }^{5,7}$

Endoscopic endonasal approaches (EEAs) to the petrous apex have been used with increasing frequency. The safety and efficacy of the EEA have been demonstrated in multiple publications. ${ }^{1,4,7}$ Medial petrous apex lesions extending into the sphenoid sinus can be accessed directly with minimal risk to the ICA via a transsphenoidal approach. Lesions of the superior petrous apex that are positioned more laterally, however, require exposure and displacement of the paraclival portion of the ICA. The inferior petrous apex can be accessed utilizing a transpterygoid infrapetrous approach. This approach requires sacrifice of the vidian nerve and introduces risk to the eustachian tube when working below the plane of the petrous segment of the ICA.

The purpose of this paper is to review the anatomy and feasibility of adding a contralateral transmaxillary (CTM) corridor to provide a wider angle of approach to the pe-

ABBREVIATIONS CTM = contralateral transmaxillary; EEA = endoscopic endonasal approach; GTR = gross-total resection; ICA = internal carotid artery. SUBMITTED September 28, 2016. ACCEPTED April 28, 2017.

INCLUDE WHEN CITING Published online October 20, 2017; DOI: 10.3171/2017.4.JNS162483. 


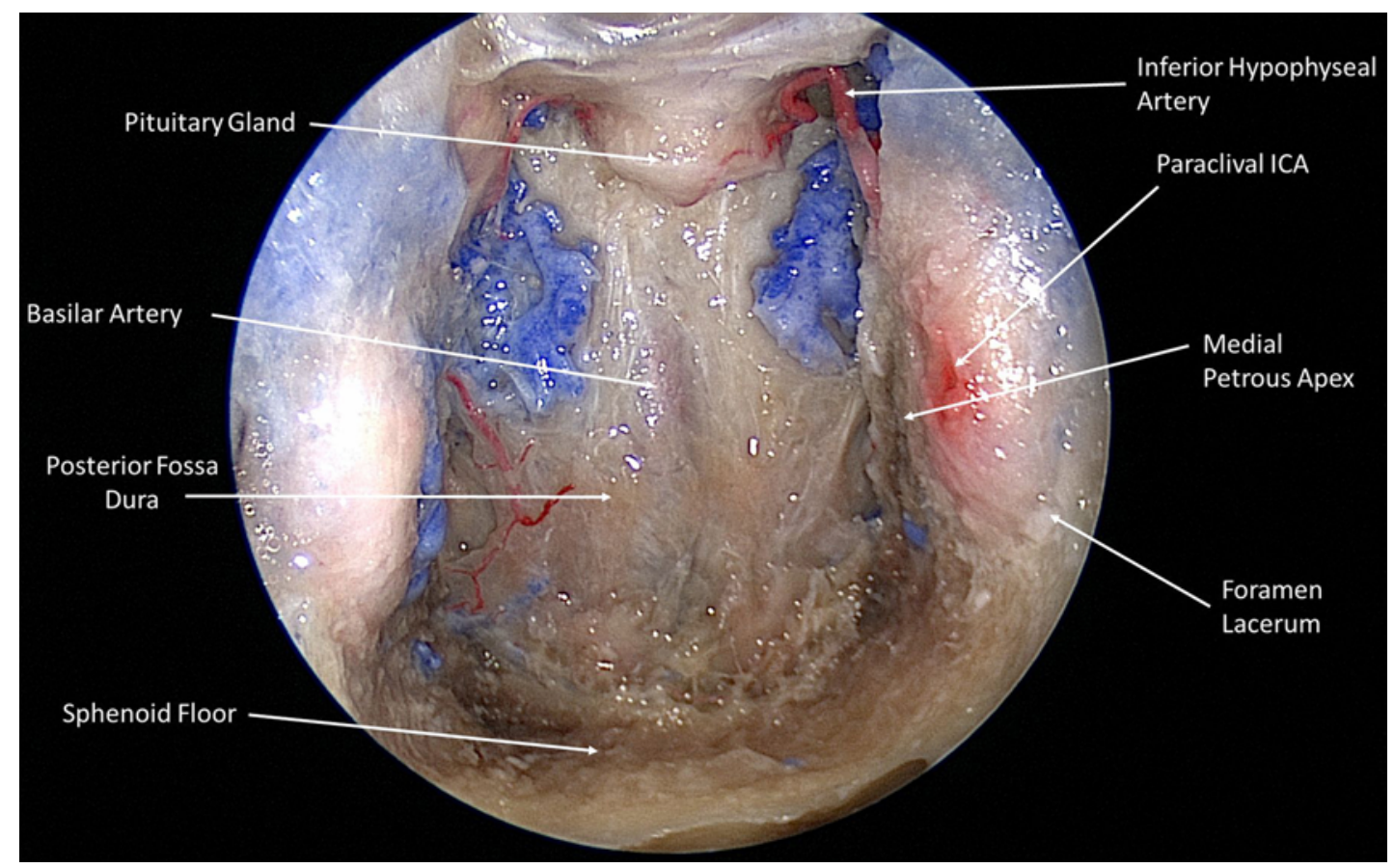

FIG. 1. Anatomical dissection of an endonasal approach to the petrous apex. An extended dissection has been performed to demonstrate key anatomical structures and landmarks. Copyright Carl Snyderman. Published with permission. Figure is available in color online only.

trous apex along the axis of the petrous ICA. This could improve lateral access without the need for manipulation of the paraclival ICA.

\section{Methods}

Five human anatomical specimens (5 heads, 10 petrous apices) were prepared for dissection at the Surgical Neuroanatomy Lab at the University of Pittsburgh. The use of human cadaveric specimens for anatomical studies has been approved by the Committee for Oversight to Research Involving the Dead (CORID). The common carotid arteries, vertebral arteries, and internal jugular veins were dissected, isolated, and cannulated with flexible tubing. Each vessel was flushed to remove blood clots with approximately $500 \mathrm{ml}$ of warm water in two separate sessions. The vessels were injected with $50 \mathrm{ml}$ of a polymethyl siloxane/silicone conglomerate, in a 2:1 preparation for arteries and 1:1 for veins, and dyed with red or blue water-soluble pigments, respectively. Dilaurate calcium carbonate $(5 \mathrm{ml})$ was added as a catalyst immediately before infusion. Specimens were refrigerated overnight and then preserved in 200 proof ethyl alcohol diluted in water as a $70 \%$ solution. Five screws were placed, distributed in the cranial area, and high-resolution CT scanning with $1 \mathrm{~mm}$ of separation acquisition was performed per neuronavigation protocol (Stryker).

Each head was stabilized with a Mayfield head holder simulating the surgical position obtained in the operating room. Endonasal approaches were performed in all 5 heads (10 sides) under endoscopic visualization (Hopkins II, Karl Storz; $4 \mathrm{~mm}$ by $18 \mathrm{~cm}, 0^{\circ}$ and $45^{\circ}$ ). Approaches began with resection of both middle turbinates followed by bilateral maxillary antrostomies. Total ethmoidectomies were also performed to achieve wider visualization for the sake of photodocumentation. Bilateral sphenoidotomies, posterior septectomy, and resection of the sphenoid rostrum were then performed to provide wide binarial exposure. To standardize angle measurements given varying degrees of pneumatization, bone over the medial paraclival ICA was thinned using the drill until the vessel was visible through a thin shell of bone (Fig. 1).

Caldwell-Luc procedures (anterior maxillotomies) were then performed bilaterally to open the contralateral transmaxillary corridor. The maxillotomy was maximally widened to the zygomatic buttress and maxillary sinus wall laterally, to the infraorbital nerve and foramen superiorly, with preservation of the nasomaxillary buttress medially and without injury to the dental roots inferiorly (Fig. 2).

At this point, an image guidance probe was passed through the nasal cavity and rested on the bone of the contralateral medial petrous apex in various positions. Screen captures of the image guidance software were taken. The nasal pointer was then passed using the CTM corridor toward the petrous apex. In most specimens, there was a limitation in the degree of retraction of soft tissues of the cheek, which increased the inferosuperior angle of approach. This required enlargement of the maxillary antrostomy with a partial medial maxillectomy to allow access for the nasal pointer. Once the pointer was able to comfortably reach the medial petrous apex, additional screen captures were obtained. This was performed bilaterally in all 5 head specimens (Fig. 3). 


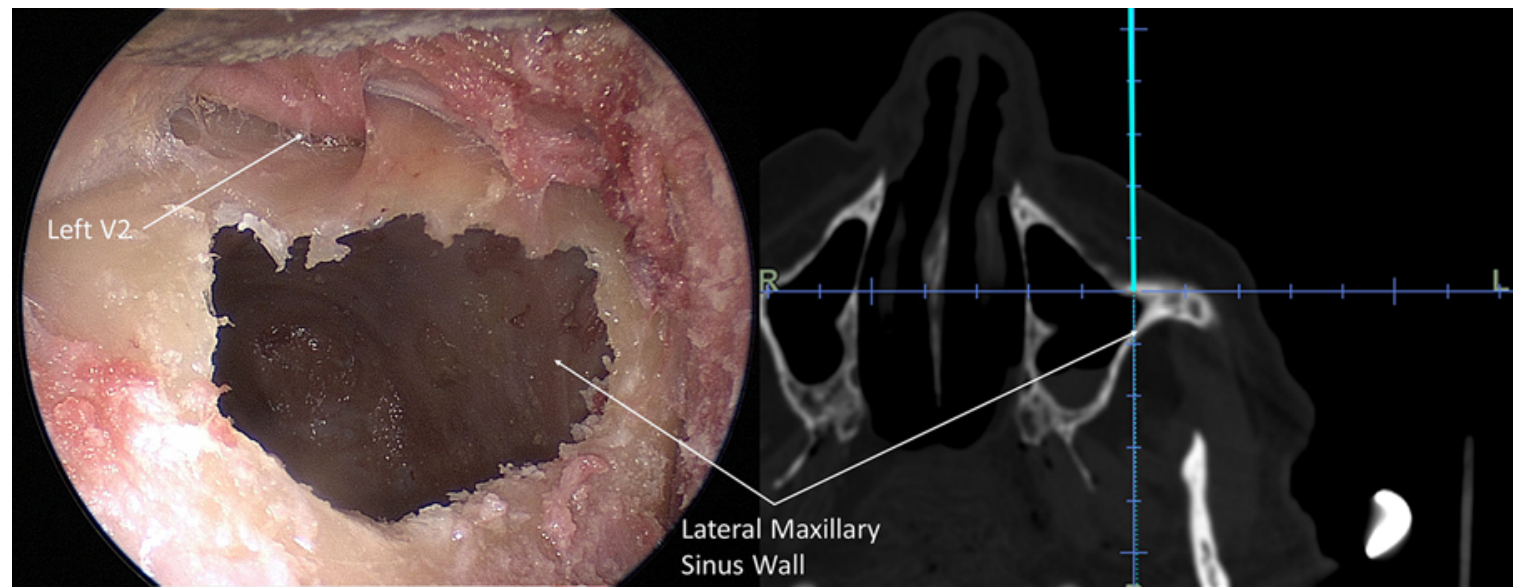

FIG. 2. Left: Anterior view of face of maxillary sinus after anterior and medial maxillotomies. Right: Axial CT scan from navigation software showing the lateral extent of anterior maxillotomy. Left panel copyright Carl Snyderman. Published with permission. Figure is available in color online only.

Screen captures were then analyzed using the GNU Image Manipulation Program (GIMP), a freely distributed piece of software. The angle of approach with respect to the axis of the petrous ICA was measured on each side
(Fig. 4). Multiple images were analyzed for each approach and the largest values were recorded. These data were recorded and analyzed in Microsoft Excel (Microsoft Corp., Redmond, WA).
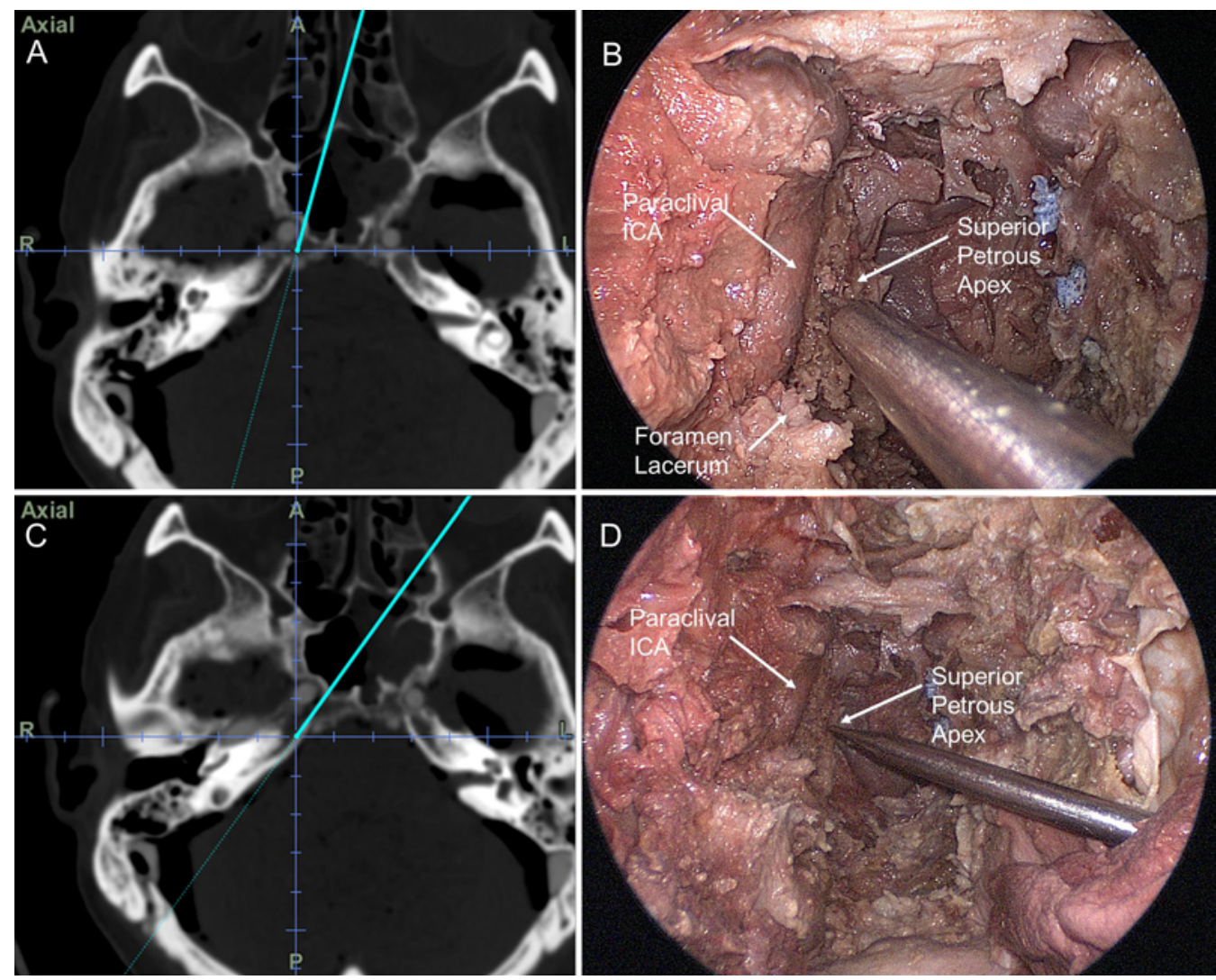

FIG. 3. A: Axial CT scan from navigation software showing the standard endonasal trajectory to the right superior petrous apex. B: Endoscopic view of the standard endonasal approach to the right petrous apex. C: Axial CT scan from navigation software showing the CTM trajectory to the right superior petrous apex. D: Endoscopic view of the left CTM approach to the right petrous apex. Panels B and D copyright Carl Snyderman. Published with permission. Figure is available in color online only. 

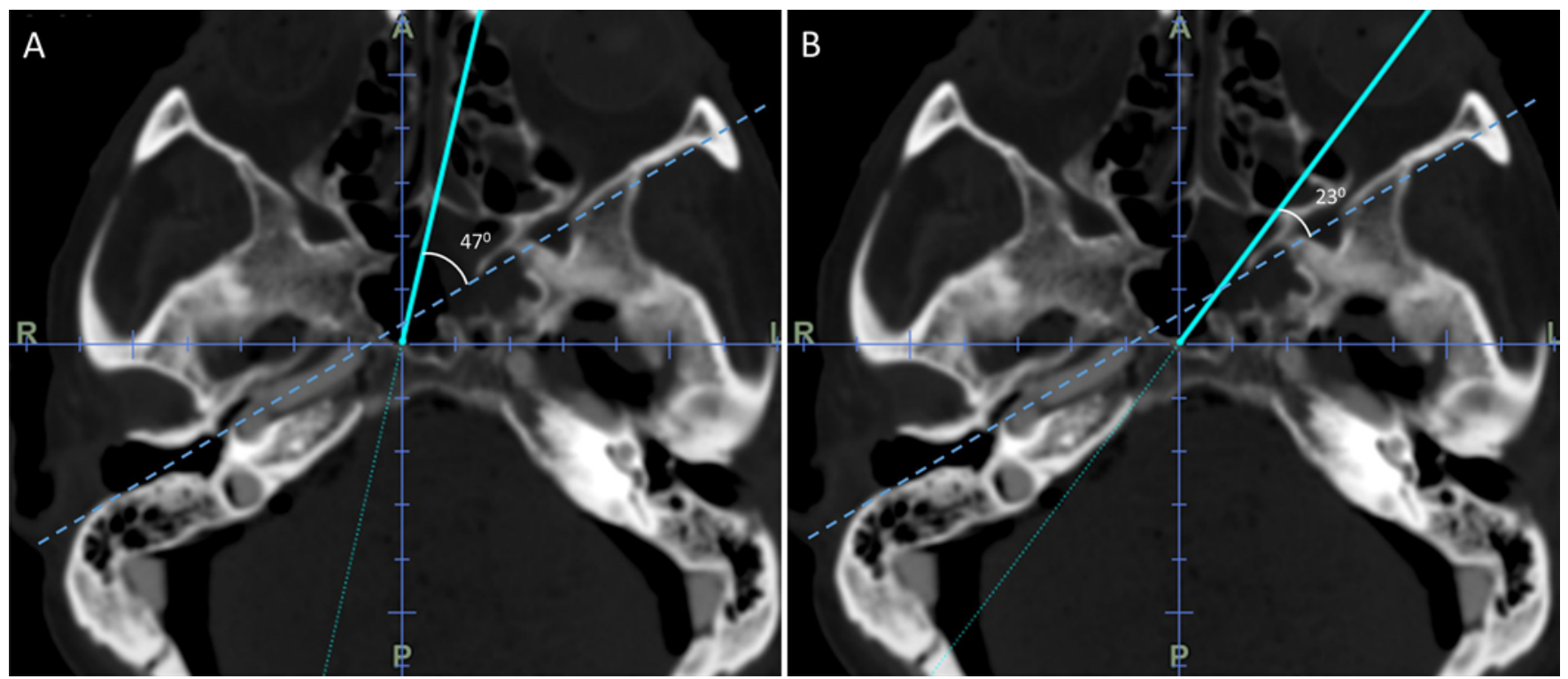

FIG. 4. Illustrative view of measurements being taken of the angle of approach with respect to the axis of the petrous ICA for the endonasal approach (A) and the CTM approach (B). The dashed line represents the axis of the petrous ICA. Figure is available in color online only.

\section{Results}

With respect to the axis of the petrous ICA (Table 1), the CTM corridor allowed for a more parallel trajectory. The endonasal approach had a mean angle of $44.8^{\circ} \pm 2.78^{\circ}$ off the axis of the petrous ICA. The CTM corridor decreased this to $20.1^{\circ} \pm 4.31^{\circ}$, a decrease of $24.7^{\circ} \pm 2.58^{\circ}$ (Fig. 5). The CTM corridor allowed the drill to access areas of the petrous apex posterior and lateral to the paraclival ICA that were not accessible by the endonasal route without extensive dissection and mobilization of the ICA (Fig. 6). Only one cadaver required opening of the pterygopalatine fossa and some drilling of the pterygoid base ipsilateral to the maxillotomy to obtain adequate access. Sacrifice of the vidian nerve was not necessary. This allowed for preservation of the sphenopalatine artery and thus the nasal

TABLE 1. Measurements in degrees of the angle of approach with respect to the axis of the petrous ICA

\begin{tabular}{ccccc}
\hline \multirow{2}{*}{$\begin{array}{c}\text { Specimen } \\
\text { No. }\end{array}$} & Side & Transnasal & CTM & \begin{tabular}{c} 
Difference \\
\cline { 3 - 4 }$\left({ }^{\circ}\right)$
\end{tabular} \\
\hline 1 & $\mathrm{Rt}$ & 47 & 23 & 24 \\
\hline 1 & $\mathrm{Lt}$ & 44 & 18 & 26 \\
\hline 2 & $\mathrm{Rt}$ & 48 & 26 & 22 \\
\hline 2 & $\mathrm{Lt}$ & 50 & 27 & 23 \\
\hline 3 & $\mathrm{Rt}$ & 43 & 20 & 23 \\
\hline 3 & $\mathrm{Lt}$ & 45 & 22 & 23 \\
\hline 4 & $\mathrm{Rt}$ & 44 & 16 & 28 \\
\hline 4 & $\mathrm{Lt}$ & 41 & 18 & 23 \\
\hline 5 & $\mathrm{Rt}$ & 42 & 17 & 25 \\
\hline 5 & $\mathrm{Lt}$ & 44 & 14 & 30 \\
\hline
\end{tabular}

septal flap vasculature ipsilateral to the maxillotomy in 4 of the 5 specimens.

A summary of our illustrative cases is provided in Table 2 .

\section{Illustrative Case}

\section{Case 2}

A 42-year-old woman presented with recurrent clival chordoma following multiple endonasal surgeries, proton

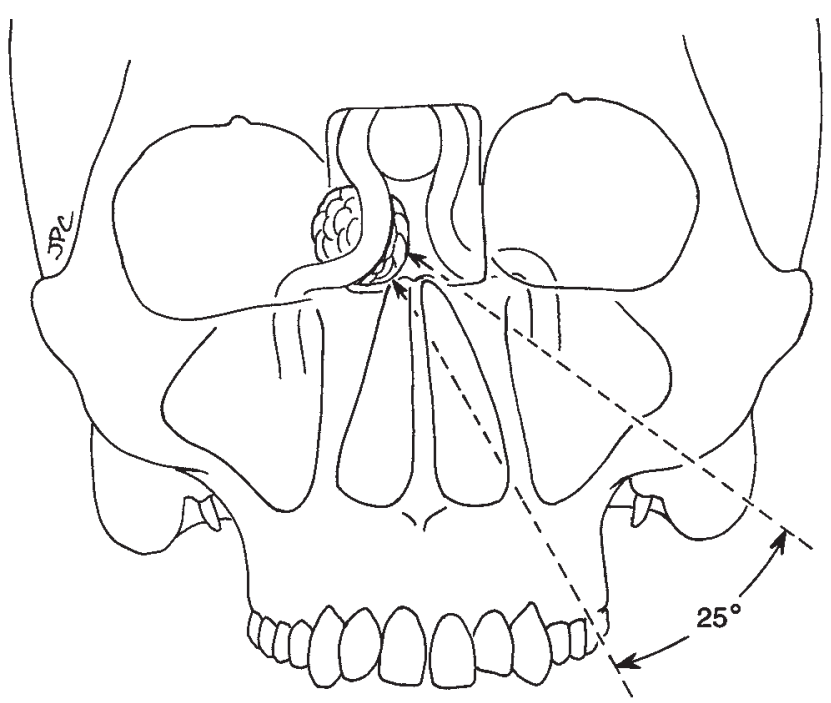

FIG. 5. Schematic drawing of a right petrous apex tumor. In comparison with an endonasal approach, the CTM approach decreases the angle of the corridor by $25^{\circ}$. Copyright Jon Coulter. Published with permission. 


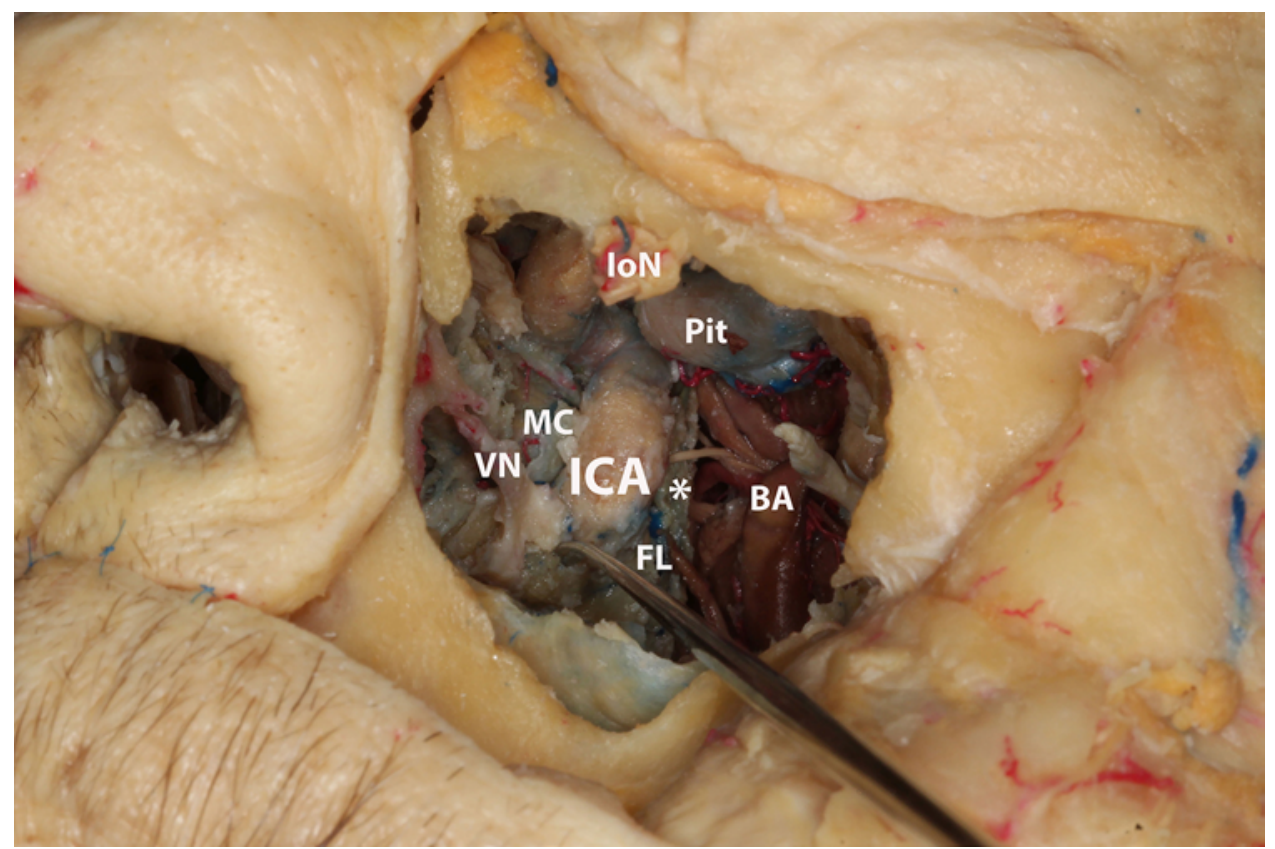

FIG. 6. Anatomical dissection demonstrating a left CTM approach to the right petrous apex. $B A=$ basilar artery; $F L=$ foramen lacerum; ICA = right paraclival portion of the ICA; IoN = infraorbital nerve; $M C=$ Meckel's cave; Pit = pituitary; VN = vidian nerve; ${ }^{*}=$ right medial petrous apex. Copyright Carl Snyderman. Published with permission. Figure is available in color online only.

beam therapy, and Gamma Knife radiosurgery (Table 2). Her symptoms at the time of recurrence included diplopia and left-sided facial hypesthesia. Her physical examination was significant for cranial nerve III, IV, and VI palsies of the left eye and decreased sensory and motor function of the left trigeminal nerve. Imaging demonstrated tumor invasion of the left cavernous sinus and Meckel's cave with encasement of the left cavernous portion of the ICA (Fig. 7). The patient underwent revision surgery, which included a right transmaxillary approach in addition to an endoscopic transclival approach to the posterior cranial fossa and an endoscopic left transpterygoid approach to the middle cranial fossa (Fig. 8). The transmaxillary approach was used to complete dissection of tumor deep to the cavernous portion of the ICA and was supplemented with dissection lateral to the ICA (Fig. 9).
Gross tumor invasion of the trigeminal nerve was noted. A gross-total resection (GTR) was achieved. The surgical site was reconstructed with fascia lata, adipose tissue, and a left extended inferior turbinate vascularized flap. The postoperative course was uncomplicated. Follow-up imaging demonstrated a GTR without evidence of recurrence (Fig. 10). Postoperatively, nasal endoscopy revealed a well-healed surgical site with bilateral openings into the maxillary sinuses (Fig. 11).

\section{Discussion}

Endoscopic endonasal approaches have greatly improved our access to the medial aspects of the petrous apex. The ability to extend endonasal approaches laterally is limited most notably by the ICA. Current techniques

TABLE 2. Clinical series of patients in whom the CTM approach was used

\begin{tabular}{|c|c|c|c|c|c|c|}
\hline Case No. & Age (yrs) & Diagnosis & Location & Approaches & Margins & Flap \\
\hline 1 & 63 & Chordoma & $\begin{array}{l}\text { Clivus, rt clCA, Dorello's } \\
\text { canal, Meckel's cave }\end{array}$ & Transclival; rt transpterygoid; It CTM & GTR & $\begin{array}{l}\text { Rt inferior turbinate } \\
\text { flap }\end{array}$ \\
\hline 2 & 42 & Chordoma & $\begin{array}{l}\text { Clivus, It clCA, Meckel's } \\
\text { cave }\end{array}$ & Transclival; It transpterygoid; rt CTM & GTR & $\begin{array}{l}\text { Lt inferior turbinate } \\
\text { flap }\end{array}$ \\
\hline 3 & 53 & Chordoma & $\begin{array}{l}\text { Clivus, rt pICA, BA, rt FR, } \\
\quad \text { rt FO }\end{array}$ & Transclival; rt transpterygoid; It CTM & $\begin{array}{l}\text { STR (GTR of } \\
\text { petrous apex) }\end{array}$ & Lt extended NSF \\
\hline 4 & 67 & Chordoma & Clivus, rt petrous apex, BA & Transclival, It CTM & GTR & Rt extended NSF \\
\hline 5 & 71 & Chondrosarcoma & Clivus, rt petrous apex & Transclival; rt transpterygoid; It CTM & GTR & Lt extended NSF \\
\hline
\end{tabular}

$\mathrm{BA}=$ basilar artery; $\mathrm{CICA}$ = cavernous portion of the ICA; FO = foramen ovale; $F R=$ foramen rotundum; NSF = nasoseptal flap; pICA = paraclival portion of the ICA; STR = subtotal resection. 

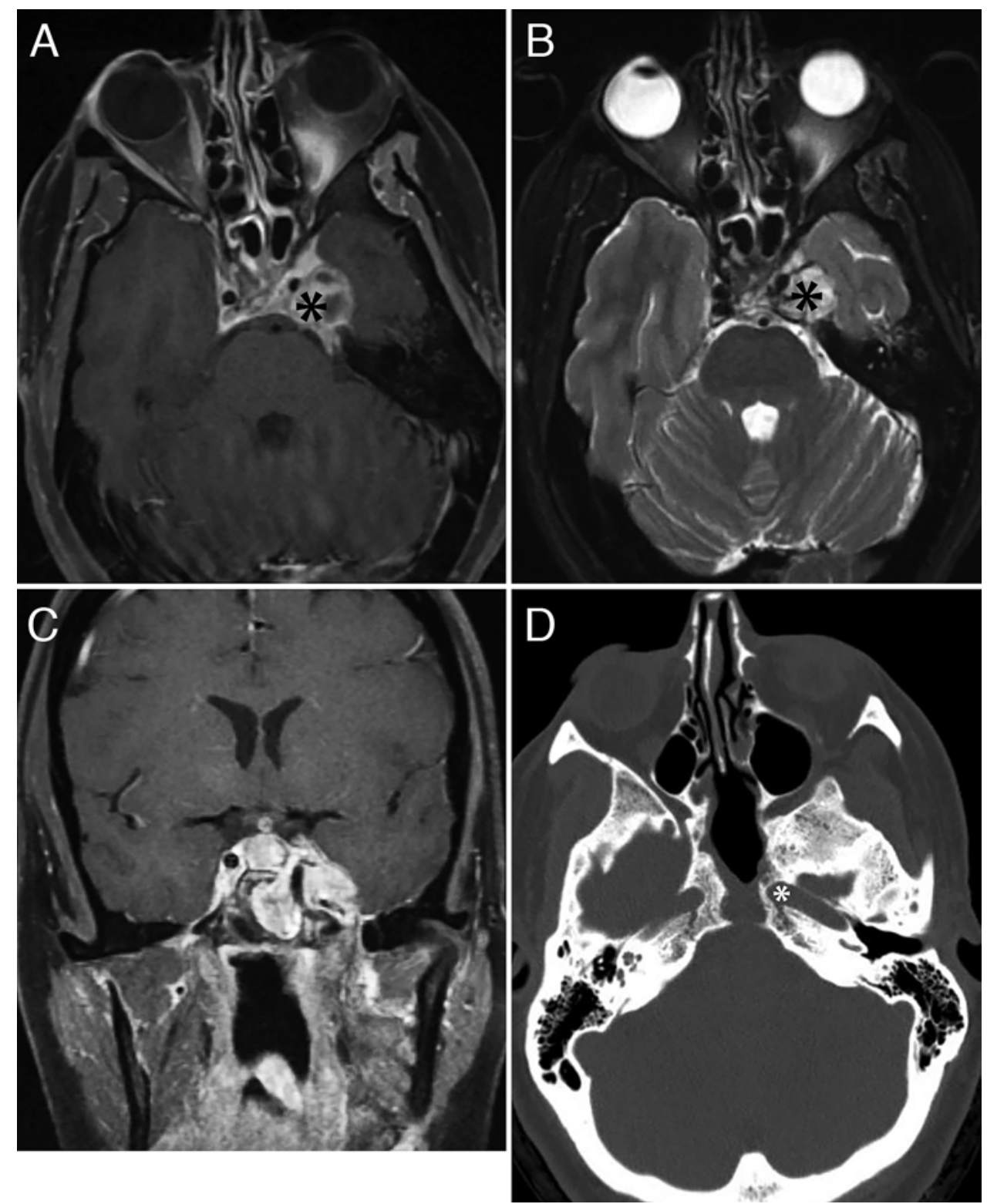

FIG. 7. Case 2. Preoperative imaging studies. A: Axial T1-weighted contrast-enhanced MR image demonstrating recurrent chordoma of the left petrous apex (asterisk). B: Axial T2-weighted MR image showing recurrent chordoma of the left petrous apex (asterisk). C: Coronal T1-weighted contrast-enhanced MR image demonstrating recurrent chordoma encasing the left paraclival ICA. D: Axial CT scan of prior surgical defect showing intact bone over second genu of the ICA (asterisk).

to circumvent this barrier include skeletonizing and lateralizing the paraclival ICA for superiorly based lesions, or working inferior to the petrous ICA for inferior ones. However, these techniques introduce significant risk for vascular injury as well as morbidity to the vidian nerve, eustachian tube, and potentially the maxillary division of the trigeminal nerve $\left(\mathrm{V}_{2}\right) \cdot{ }^{5,7}$

The most common lesion of the petrous apex is a cholesterol granuloma. Multiple studies have shown comparable outcomes with the EEA compared with lateral approaches but with a lower morbidity rate. ${ }^{1}$ Complication rates are lower with the endonasal route, and the severity of those complications, as well as their impact on quality of life, is lower. Large expansive cholesterol granulomas can be effectively drained without manipulation of the ICA in most cases, but the ability to dissect within the cavity is limited without displacement of the paraclival portion of the ICA.

Large clival chordomas and chondrosarcomas that extend laterally are also difficult to manage endonasally. Koutourousiou et al. ${ }^{3}$ recently reported that chordomas with significant lateral extension often required a second open approach, were less likely to be gross totally resected, and thus had a higher rate of recurrence in a shorter period of time. The ability to safely work behind the ICA endonasally may reduce the need for second procedures and improve the chances of GTR. 


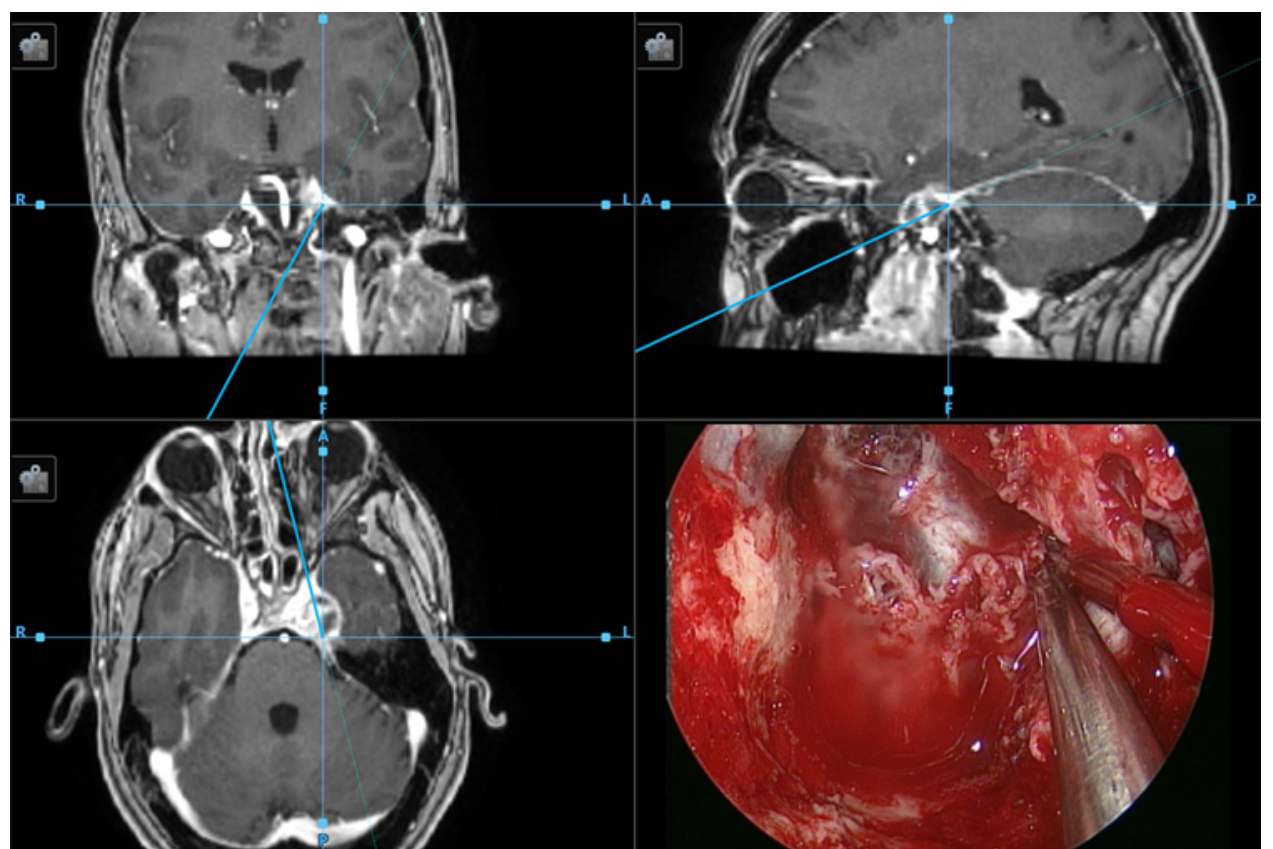

FIG. 8. Case 2. Intraoperative navigation screen captures demonstrating the endonasal angle of approach. The probe is displacing the paraclival ICA. Figure is available in color online only.

The difficulty in accessing the more lateral aspects of the petrous apex via an EEA comes from the limited angle of approach that the endonasal route offers due to the bony confines of the pyriform aperture. A similar limitation is encountered when attempting to reach the infratemporal fossa endonasally. Two techniques that are employed to overcome this barrier are Denker's anteromedial maxillotomy and the anterior maxillotomy via a Caldwell-Luc procedure. Morbidity associated with these extensions is minimal and generally limited to minor cosmetic changes. ${ }^{2,6}$

Given the efficacy of the transmaxillary corridor for the

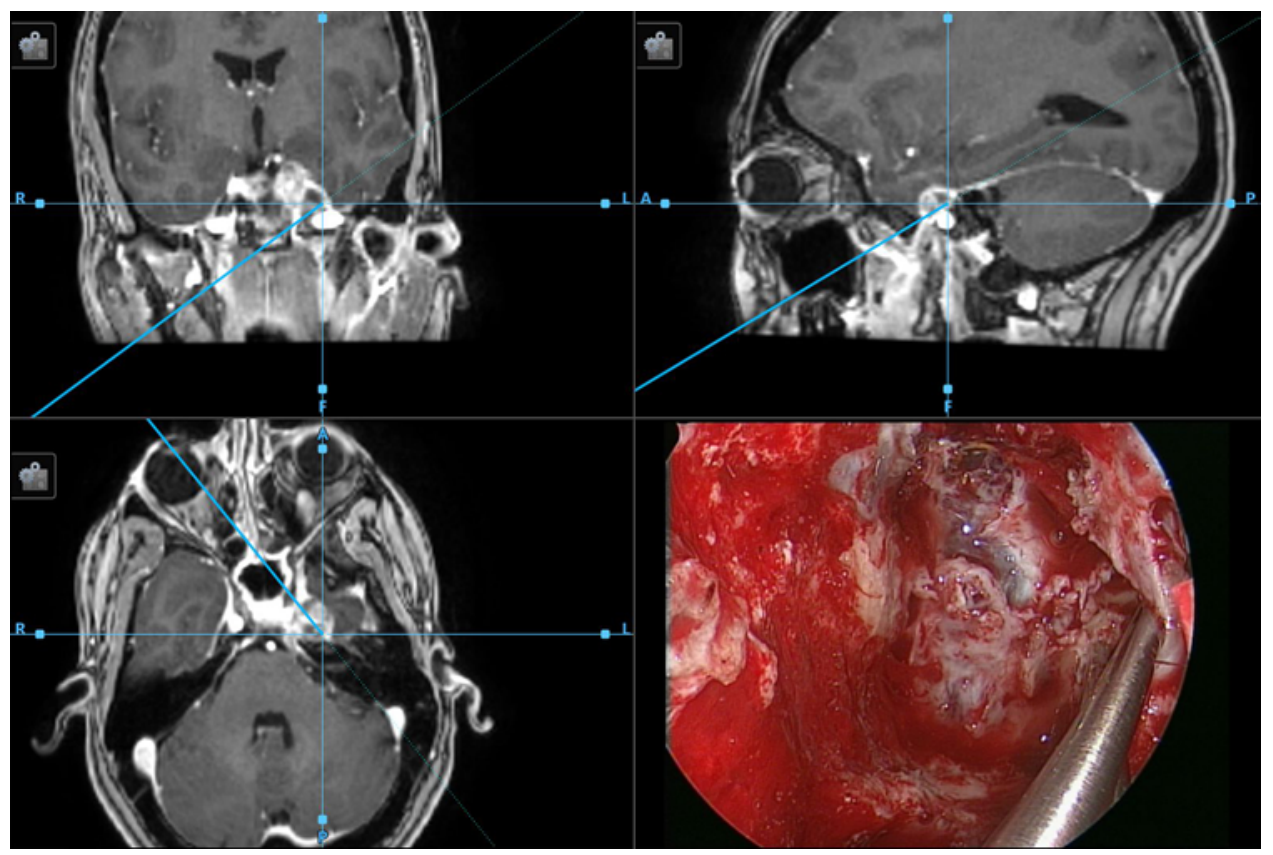

FIG. 9. Case 2. Intraoperative navigation screen captures demonstrating the angle with the CTM approach. The probe is displacing the paraclival ICA. Figure is available in color online only. 

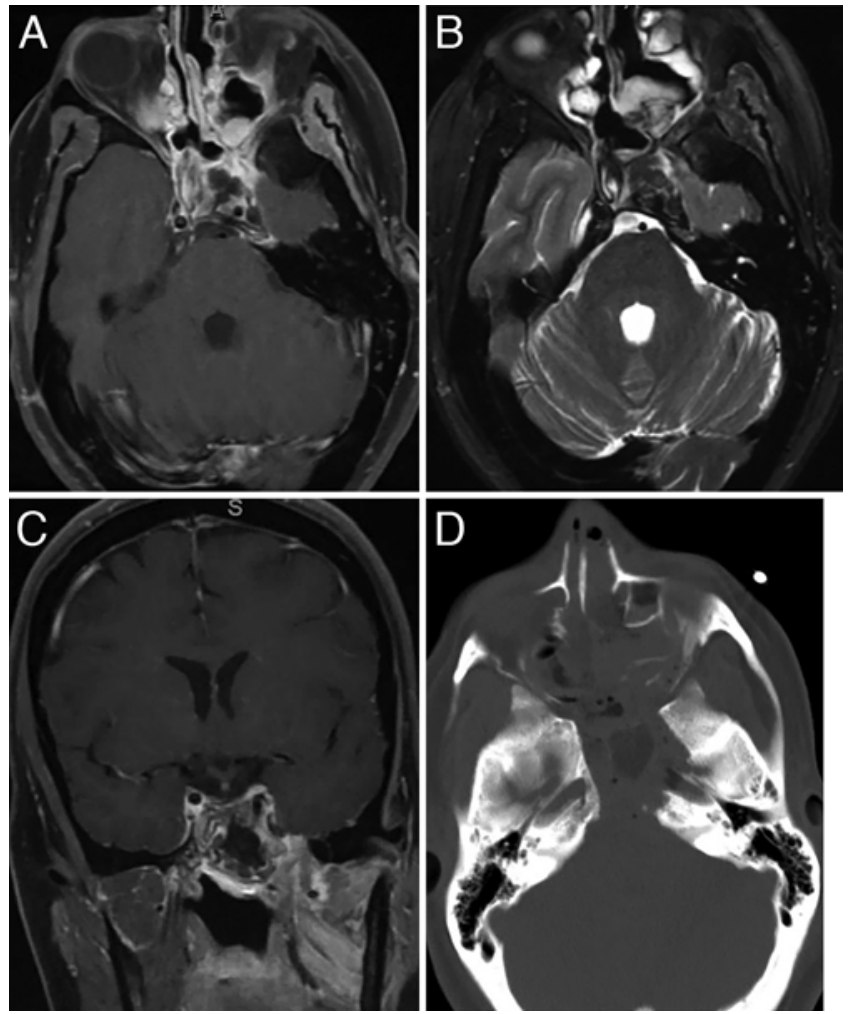

FIG. 10. Case 2. Postoperative images demonstrating a GTR of the chordoma: axial T1-weighted contrast-enhanced MR image (A); axial T2-weighted MR image (B); coronal T1-weighted contrast-enhanced MR image (C); and axial CT scan (D) demonstrating additional bone removal accomplished.

ipsilateral infratemporal fossa, we explored using the same approach to offer a wider angle of approach to the contralateral petrous apex. First, it was important to ensure that the anterior maxillotomy was taken as far laterally as possible to maximize access. In addition to the CaldwellLuc procedure, a partial resection of the medial maxillary wall was necessary on the same side as the anterior maxillotomy. The posterior third to half of the inferior turbinate was a barrier to instruments traversing the corridor, and a simple displacement of the inferior turbinate or partial medial maxillectomy was necessary in the cadaver specimens to open the pathway. This was confirmed in our illustrative cases where medial maxillectomies were necessary to maximize the access. In some cases it may also be helpful to drill the medial aspect of the base of pterygoid to improve the window of access, especially for larger instruments like drills or other powered instruments. Sacrifice of the vidian nerve was not necessary on the side of the maxillotomy.

The CTM corridor increased the angle of approach relative to the paraclival ICA by $24.7^{\circ} \pm 2.58^{\circ}$, allowing significantly more access to the area of the petrous apex posterior to the ICA. The wider the window between the ICA and the posterior fossa dura, the further lateral we were able to reach. More importantly, this access was obtained without requiring a complete exposure and mobilization of the ICA in all cases. It is still necessary to

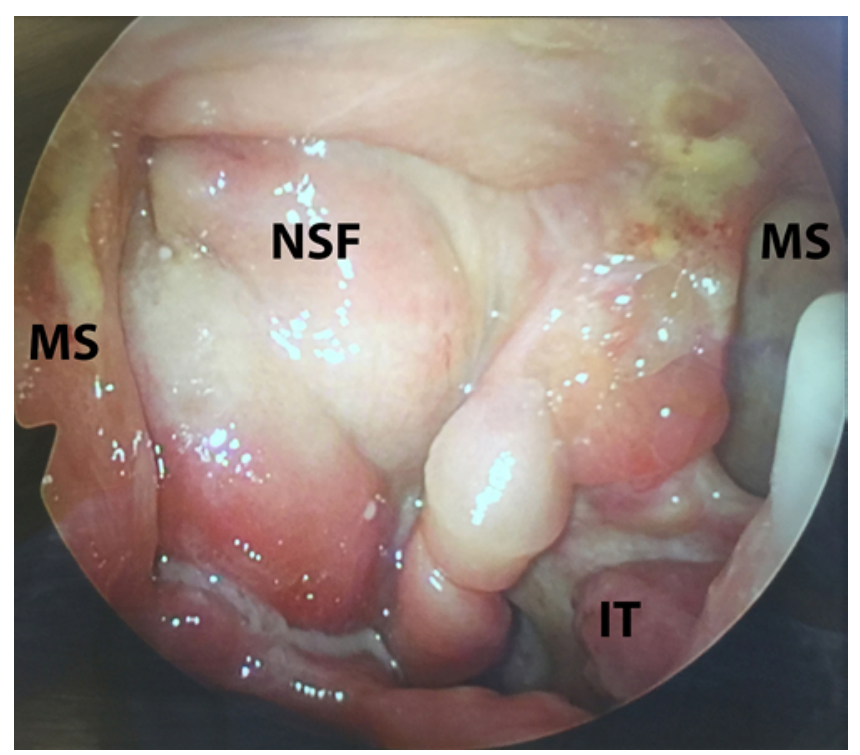

FIG. 11. Postoperative nasal endoscopy of the right nasal cavity demonstrates a right CTM approach to the left petrous apex. The nasoseptal flap covers the posterior fossa defect, left petrous apex, and paraclival ICA. IT = inferior turbinate; $M S$ = maxillary sinus; NSF = nasoseptal flap reconstruction. Figure is available in color online only.

thin the bone over the paraclival ICA to clearly identify it and understand its location during dissection. However, by preserving a covering of bone and avoiding manipulation, the risk of vascular injury is minimized. Combined with intraoperative navigation, we feel this strategy can markedly improve the safety of extended endonasal petrous apex approaches. Depending on the extent of disease, mobilization of the paraclival ICA may still be performed to provide additional access.

The angle of approach with respect to the axis of the horizontal petrous ICA was also markedly reduced, providing a corridor more parallel to the petrous ICA. This enhances the ability of the surgeon to visualize and dissect the inferior and posterior aspects of the petrous apex. As a result, the CTM corridor may obviate the need for a full transpterygoid approach with sacrifice of the vidian nerve and eustachian tube. Passage of instruments is also improved due to the straight corridor and direct visualization with a $0^{\circ}$ endoscope. Blind instrumentation behind the ICA could be avoided.

The CTM corridor preserves reconstructive options with the nasal septal flap. Generally, if a reconstructive flap is necessary, it is placed on the side opposite the lesion because a transpterygoid approach is necessary ipsilateral to the lesion to reach laterally. With a CTM approach, the transpterygoid approach may not be necessary, and the vasculature on that side may be preserved. It would be advantageous to place the flap on the side ipsilateral to the lesion as it would increase the reach of the flap and potentially improve coverage. This is also advantageous because it may be necessary to sacrifice the vasculature on the side ipsilateral to the maxillotomy to gain full access (via pterygoid base drilling) with a CTM approach. However, the pedicle ipsilateral to the maxillotomy can be 
preserved as well by unroofing the pterygopalatine fossa and mobilizing the pedicle to the pterygoid canal.

We have presented our initial clinical experience with this approach on 4 recurrent clival chordomas that were made exceedingly difficult by their recurrent nature, prior surgery and radiotherapy, and extensive lateral extension. The fifth case was a low-grade chondrosarcoma with significant displacement of the petrous ICA. The CTM corridor allowed for improved lateral access and was helpful in achieving our surgical goals. A full mobilization of the ICA with complete bony decompression and transection of the cartilage of the foramen lacerum achieves similar results and is an alternative to the approach but introduces considerable risk of carotid artery injury. However, by minimizing the extent of manipulation of the ICA with the CTM corridor, we feel the risk of vascular injury is decreased, especially in the demonstrated setting, where scarring from prior surgery and radiation-induced changes can significantly increase this risk.

While this technique improves lateral access, it is critical to carefully review imaging studies for each case and consider alternative approaches based on the diagnosis, anatomical variations, and goals of surgery. A transpterygoid infrapetrous approach or open lateral approach may be preferable for certain cases, particularly for very inferior and lateral lesions. Prior studies have shown that the most common site for residual chordoma following EEA is inferior and lateral, ${ }^{6}$ and this study has shown that the CTM corridor improves access to these areas. The greatest utility of the CTM approach is likely for less expansive cholesterol granulomas and clival tumors (chordoma and chondrosarcoma) that are positioned posterolateral to the ICA.

\section{Conclusions}

The CTM corridor is a useful adjunct to previously described EEA to the petrous apex. It offers a trajectory more parallel to the course of the horizontal petrous ICA and thereby allows greater access to more lateral aspects of the petrous apex without necessitating an extensive manipulation of the ICA. This may reduce the risk of endoscopic endonasal surgery to the petrous apex while increasing access.

\section{References}

1. Eytan DF, Kshettry VR, Sindwani R, Woodard TD, Recinos PF: Surgical outcomes after endoscopic management of cholesterol granulomas of the petrous apex: a systematic review. Neurosurg Focus 37(4):E14, 2014
2. Falcon RT, Rivera-Serrano CM, Miranda JF, Prevedello DM, Snyderman CH, Kassam AB, et al: Endoscopic endonasal dissection of the infratemporal fossa: Anatomic relationships and importance of eustachian tube in the endoscopic skull base surgery. Laryngoscope 121:31-41, 2011

3. Koutourousiou M, Gardner PA, Tormenti MJ, Henry SL, Stefko ST, Kassam AB, et al: Endoscopic endonasal approach for resection of cranial base chordomas: outcomes and learning curve. Neurosurgery 71:614-625, 2012

4. Paluzzi A, Gardner P, Fernandez-Miranda JC, Pinheiro-Neto CD, Scopel TF, Koutourousiou M, et al: Endoscopic endonasal approach to cholesterol granulomas of the petrous apex: a series of 17 patients: clinical article. J Neurosurg 116:792798, 2012

5. Scopel TF, Fernandez-Miranda JC, Pinheiro-Neto CD, PerisCelda M, Paluzzi A, Gardner PA, et al: Petrous apex cholesterol granulomas: endonasal versus infracochlear approach. Laryngoscope 122:751-761, 2012

6. Van Rompaey J, Solares CA: Transmaxillary approach to the infratemporal fossa. Oper Tech Otolaryngol 24:218-221, 2013

7. Zanation AM, Snyderman CH, Carrau RL, Gardner PA, Prevedello DM, Kassam AB: Endoscopic endonasal surgery for petrous apex lesions. Laryngoscope 119:19-25, 2009

\section{Disclosures}

The authors report no conflict of interest concerning the materials or methods used in this study or the findings specified in this paper.

\section{Author Contributions}

Conception and design: Snyderman, Patel. Acquisition of data: Snyderman, Patel, Gardner. Analysis and interpretation of data: Snyderman, Patel, Wang, Gardner. Drafting the article: Snyderman, Patel, Gardner. Critically revising the article: all authors. Reviewed submitted version of manuscript: all authors. Administrative/technical/material support: Wang. Study supervision: Snyderman, Gardner.

\section{Supplemental Information}

\section{Previous Presentations}

Portions of this work were presented in poster form at the 2016 Annual Meeting of the North American Skull Base Society, Scottsdale, AZ, February 12-14, 2016.

\section{Correspondence}

Carl Snyderman, UPMC Center for Cranial Base Surgery, The Eye \& Ear Institute, 200 Lothrop St., Ste. 500, Pittsburgh, PA 15213. email: snydermanch@upmc.edu. 